Kompass

Autoimmun

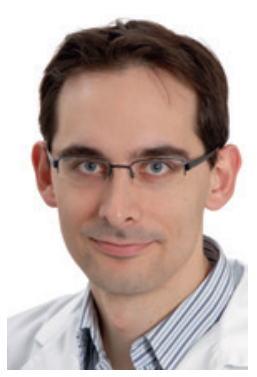

\title{
CED - Erkrankungsaktivität und Steroidtherapie als Risikofaktoren für COVID-19?
}

Bertram Bengsch

Klinik für Innere Medizin II Gastroenterologie, Hepatologie, Endokrinologie und Infektiologie, Universitätsklinikum Freiburg, Freiburg, Deutschland

Zusammenfassung zu Lukin DJ, Kumar A, Hajifathalian K, et al.: Baseline disease activity and steroid therapy stratify risk of COVID-19 in patients with inflammatory bowel disease. Gastroenterology. 2020;DOI:10.1053/j.gastro.2020.05.066.

\section{Keywords}

COVID-19 $\cdot$ IBD · SARS-CoV-2 · Biologic therapy

\section{Zusammenfassung}

Die durch das SARS-CoV-2 Virus verursachte Pandemie und COVID-19-Erkrankung stellen weltweit die Gesundheitssysteme vor große Herausforderungen. Es bestehen große Unsicherheiten, ob Patientengruppen mit autoimmun-vermittelten Vorerkrankungen und immunsuppressiven Therapien ein höheres Risiko für ungünstigere Verläufe einer COVID-19-Erkrankung haben. Um den Einfluss von COVID-19 auf Patienten mit chronischentzündlichen Darmerkrankungen (CED) zu untersuchen, haben Lukin et al. in einer aktuellen Arbeit in Gastroenterology in der stark betroffenen Metropole New York zum einen eine Fall-Kon- trollstudie durchgeführt, um die klinischen Verläufe von CED-Patienten, die an COVID-19 erkrankt waren, zu untersuchen, sowie eine longitudinale Analyse in einer definierten Kohorte von CEDPatienten mit aktiver Erkrankung vorgenommen, um den Einfluss der CED-Erkrankungsaktivität und des Therapieregimes auf das Risiko einer COVID-19-Erkrankung zu prüfen.

Die Ergebnisse der Untersuchungen legen nahe, dass CED-Patienten kein erhöhtes Risiko für einen schweren Verlauf von COVID-19 haben. Patienten mit Colitis ulcerosa waren etwas stärker betroffen als Patienten mit Morbus Crohn. COVID-19 führte bei CED-Patienten zu verstärkter gastrointestinaler Symptomatik. Patienten mit aktiver CED und Steroidtherapie waren häufiger von COVID-19 betroffen als Patienten mit Biologikatherapie.

c) 2020 S. Karger GmbH, Freiburg 


\section{Transfer in die Praxis}

\section{Hintergrund}

Die durch das SARS-CoV-2-Virus hervorgerufene COVID-19-Pandemie stellt die Gesundheitssysteme weltweit vor ausgeprägte Herausforderungen. Gerade bei Patienten mit immunsuppressiver Therapie ist unklar, ob die Immunsuppression allgemein oder bestimmte Immunsuppressiva speziell zu einem veränderten Erkrankungsverlauf beitragen. Diese Frage ist insbesondere für Patienten mit chronisch-entzündlichen Darmerkrankungen (CED) relevant, die für einen Remissionserhalt eine immunsuppressive Dauertherapie benötigen.

In der getrennt von den oben dargestellten Auswertungen longitudinal untersuchten Kohorte von 119 an die Zentren angebundenen CED-Patienten mit aktiver Erkrankung wurde bei 29 (24\%) die Diagnose COVID-19 gestellt, was in etwa den Schätzungen für die gesamte New Yorker Bevölkerung entspricht. Bauchschmerzen und Diarrhöen waren wie in der vorhergehenden Untersuchung mit einer COVID-19-Infektion assoziiert. Diese Befunde passten zu erhöhten Entzündungsmarkern im Labor (CRP), Stuhl (Calprotectin) sowie der endoskopischen Diagnostik. Die von COVID-19 betroffenen Patienten hatten zudem signifikant häufiger eine vorbestehende Steroidtherapie. Bei 83 Patienten wurden Biologika eingesetzt, hier zeigte sich ein Trend zu niedrigeren COVID-19-Fallzahlen bei Patienten mit Ustekinumab oder TNF-Antagonisten und zu etwas höheren Zahlen bei Vedolizumab und Tofacitinib, ohne eine statistische Signifikanz zu erreichen.

\section{Ergebnisse der Studie}

Für die Studie wurde zunächst eine Kohorte herangezogen, in die zuvor 1059 Patienten mit der Diagnose einer COVID-19-Erkrankung an zwei Krankenhäusern in Manhattan eingeschlossen wurden. In dieser Kohorte wurde zuvor bereits eine hohe Prävalenz von gastrointestinalen Symptomen beschrieben [1]. In der aktuellen Arbeit wurden nun 160 COVID-19-Patienten mit 80 Patienten mit COVID-19, die zusätzlich eine CED aufwiesen, hinsichtlich Lebensdekade und Geschlecht gematcht, und in Bezug auf klinische Manifestationen der COVID-19-Erkrankung, Notwendigkeit einer intensivmedizinischen Therapie, Intubation und Tod untersucht.

Hierbei zeigte sich, dass CED-Patienten mit COVID-19 deutlich häufiger gastrointestinale Beschwerden wie Diarrhöen und abdominale Schmerzen aufwiesen. Der kombinierte Endpunkt Tod, Intensivtherapie oder Intubation wurde bei CED-Patienten etwas seltener (24\% i.v. zu 35\%) festgestellt, die Unterschiede waren aber nicht statistisch signifikant. Patienten mit Colitis ulcerosa schienen gemessen an den Vorstellungen in der Notaufnahme und der stationären Aufnahmenotwendigkeit in einer multivariaten Analyse klinisch schwerer betroffen als Patienten mit M. Crohn. Generell wiesen die CED-Patienten im Vergleich zu der Kontrollgruppe einen geringeren Body-Mass-Index (BMI) und weniger respiratorische Vorerkrankungen wie COPD oder Asthma auf, allerdings eine höhere Prävalenz von malignen Vorerkrankungen und sie nahmen häufiger eine immunsuppressive Therapie ein.
Für 64 der 80 CED-Patienten lagen Informationen zur CED-Therapie vor. Bei einem Drittel der Patienten wurden keine Biologika eingesetzt, 16 Patienten wurden mit TNF-Antikörpern, 10 Patienten mit Vedolizumab und 12 Patienten mit Ustekinumab behandelt. Es zeigte sich, dass etwa ein Drittel der Patienten ohne Biologika-Therapie oder unter Vedolizumab stationär aufgenommen werden mussten, wohingegen dies nur bei je einem Patienten mit TNFAntagonisten und Ustekinumab beobachtet wurde. Die insgesamt kleinen Fallzahlen erlauben zwar keine statistisch signifikante Aussage, weisen aber tendenziell darauf hin, dass Patienten mit Biologika-Therapie kein erhöhtes Risiko für eine schwere COVID-19-Erkrankung haben und eventuell sogar einen etwas günstigeren Verlauf nehmen könnten.

\section{Fazit für die Praxis}

Zusammengefasst weist diese erste Arbeit bei beachtlichen Fallzahlen, bei jedoch in weiteren Untersuchungen zu bestätigenden Ergebnissen, auf drei wesentliche Punkte für CED-Patienten in Bezug auf die COVID-19-Therapie hin:

1. CED-Patienten sind eher seltener von schweren Verläufen mit COVID-19 betroffen, vermutlich aufgrund der in der Kohorte typischerweise geringeren Prävalenz COVID-19-spezifischer Risikofaktoren.

2. Gastrointestinale Symptome nehmen bei Patienten mit CED im Rahmen einer COVID-19-Erkrankung deutlich zu. Die Differentialdiagnose einer SARS-CoV-2-Infektion sollte daher bei Patienten mit zunehmender oder neu aufgetretener Symptomatik evaluiert werden.

3. Immunsuppressive Therapien mit Biologika sind ausreichend sicher. In den durchgeführten Analysen zeigte sich kein erhöhtes Risiko für Patienten mit CED und Biologikatherapie. Die geringen Fallzahlen erlauben keine genaue Differenzierung zwischen den verwendeten Substanzen, waren im Gegensatz zur Steroidtherapie jedoch nicht mit der COVID-19-Infektion assoziiert. Die Daten weisen darauf hin, dass die Therapie mit Biologika auch bei relevantem Risiko für eine SARS-CoV-2-Infektion fortgeführt werden kann. Eine Steroid-sparende Therapiestrategie sollte jedoch präferiert werden.

\section{Literatur}

1 Hajifathalian K, Krisko T, Mehta A, et al.: Gastrointestinal and hepatic manifestations of 2019 novel coronavirus disease in a large cohort of infected patients from New York: clinical implications. Gastroenterology; 2020; DOI: 10.1053/j.gastro.2020.05.010.

Kontaktadresse: Prof. Dr. Dr. Bertram Bengsch, Klinik für Innere Medizin II, Gastroenterologie, Hepatologie, Endokrinologie und Infektiologie, Universitätsklinikum Freiburg, Hugstetter Straße 55, 79106 Freiburg, Deutschland, bertram.bengsch@uniklinik-freiburg.de 